\title{
Magnetocardiographic and Electrocardiographic Exercise Mapping in Healthy Subjects
}

\author{
Panu Takala, ${ }^{1,2}$ Helena Hänninen,,${ }^{2,3}$ Juha Montonen, ${ }^{1,2}$ MarkKu MäKiJärvi, ${ }^{2,3}$ JukKa Nenonen, ${ }^{1,2}$

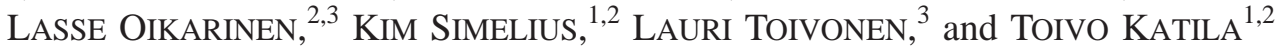 \\ ${ }^{1}$ Laboratory of Biomedical Engineering, Helsinki University of Technology, Espoo, ${ }^{2}$ BioMag Laboratory, Medical Engineering \\ Centre, and ${ }^{3}$ Division of Cardiology, Helsinki University Central Hospital, Helsinki, Finland
}

(Received 14 November 2000; accepted 13 March 2001)

\begin{abstract}
In 12-lead electrocardiography (ECG), detection of myocardial ischemia is based on ST-segment changes in exercise testing. Magnetocardiography (MCG) is a complementary method to the ECG for a noninvasive study of the electric activity of the heart. In the MCG, ST-segment changes due to stress have also been found in healthy subjects. To further study the normal response to exercise, we performed MCG mappings in 12 healthy volunteers during supine bicycle ergometry. We also recorded body surface potential mappings (BSPM) with 123 channels using the same protocol. In this paper we compare, for the first time, multichannel MCG recorded in bicycle exercise testing with BSPM over the whole thorax in middle-aged healthy subjects. We quantified changes induced by the exercise in the MCG and BSPM with parameters based on signal amplitude, and correlation between signal distributions at rest and after exercise. At the ST-segment and T-wave apex, the exercise induced a magnetic field component outward the precordium and the minimum value of the MCG signal over the mapped area was found to be amplified. The response to exercise was smaller in the BSPM than in the MCG. A negative component in the MCG signal at the repolarization period of the cardiac cycle should be considered as a normal response to exercise. Therefore, maximum ST-segment depression over the mapped area in the MCG may not be an eligible parameter when evaluating the presence of ischemia.
\end{abstract}

Keywords-Magnetocardiography, Body surface potential mapping, Exercise test, Myocardial ischemia. (C) 2001 Biomedical Engineering Society. [DOI: 10.1114/1.1376388]

\section{INTRODUCTION}

Twelve-lead exercise electrocardiography (ECG) is a well standardized and widely used diagnostic and prognostic test for evaluation of patients with ischemic heart disease. Magnetocardiography (MCG) records the magnetic field induced by the same bioelectric currents giv-

Address correspondence reprint requests to Panu Takala, LicSc, Helsinki University of Technology, Laboratory of Biomedical Engineering, P.O. Box 2200, FIN-02015 HUT, Finland. Electronic mail: panu.takala@hut.fi ing rise to the ECG. In spite of the great clinical significance of the exercise ECG, few studies on stress MCG have been reported. $1,2,4,6,8,12,17,18,22$

In principle, the MCG is more sensitive than the ECG to tangential currents in the heart. ${ }^{23}$ The MCG is also sensitive to circular vortex currents, not detectable by the ECG and it has been shown to contain information complementary to the ECG. ${ }^{2,9,16}$ In the normal heart, the main direction of the activation wave front is radial, from the endocardium to the epicardium. Thus the MCG may show deviations from the normal activation direction induced by, e.g., myocardial ischemia with better accuracy than the ECG. ${ }^{19}$ Myocardial ischemia can be detected by measuring the orientation of the magnetic field recorded in exercise MCG testing. ${ }^{8}$

Body surface potential mapping (BSPM), covering an extensive area of the thorax, offers a superior spatial resolution compared to the 12-lead ECG. The BSPM is also more sensitive than the 12-lead ECG to myocardial ischemia in coronary artery disease (CAD). ${ }^{24}$ Recordings of the BSPM and MCG in the same subjects allow a comprehensive study of magnetic and electric signals of cardiac origin.

For clinical applications, it is essential to know typical changes caused by physical exercise in the MCG of healthy subjects. Also, ST-segment changes found in the MCG but not in multilead ECG in healthy subjects during stress ${ }^{1,2}$ need further examination. In previous exercise MCG studies, BSPM has covered only a limited area of the thorax and in most studies only few subjects were included. No exercise studies combining MCG and BSPM data in middle-aged male subjects, the group with the highest risk of $\mathrm{CAD}$, have been reported. In this study, we combine multichannel MCG with BSPM recorded in exercise testing over the whole thorax. The aim was to explore the changes induced by exercise in the MCG of middle-aged healthy subjects and to compare them with changes found in the BSPM. Interindi- 


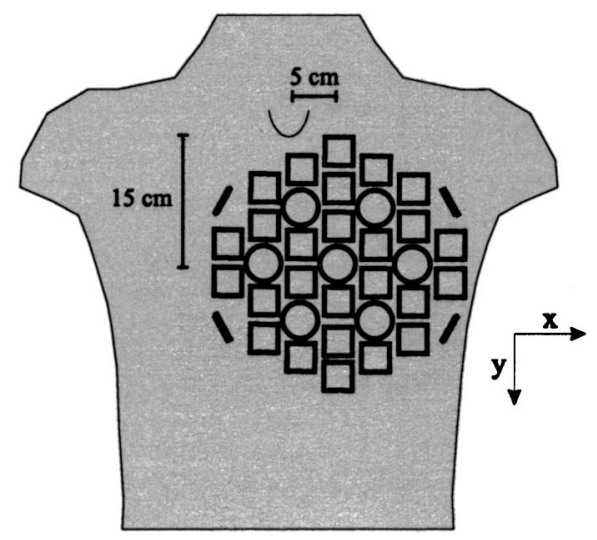

FIGURE 1. Positioning of the MCG sensor array over the chest. The circles show the locations of axial gradiometers detecting $\partial B_{z} / \partial z$, while the squares refer to planar gradiometer units detecting $\partial B_{z} / \partial x$ and $\partial B_{z} / \partial y$.

vidual variations in the MCG and BSPM signal distribution were also quantified and compared.

\section{METHODS}

Subjects

The study group comprised 12 healthy volunteers (11 males, 1 female) with the mean age of 54 years (range 45-64 years). None of the subjects had a history of cardiovascular disease. In all subjects, the standard exercise ECG and transthoracic echocardiography were normal. All subjects gave informed consent for the study.

\section{Exercise Measurements}

Exercise MCG and BSPM measurements were performed in the magnetically shielded room (MSR) of the BioMag Laboratory at the Helsinki University Central Hospital. ${ }^{14}$ Magnetocardiograms were recorded using a 67-channel cardiomagnetometer with seven coaxial and 60 planar first-order dc-superconducting quantum interference device (SQUID) gradiometers. ${ }^{11}$ The sensors are placed in a hexagonal array (Fig. 1) on a slightly curved bottom of a Dewar with an inner radius of $30 \mathrm{~cm}$. In the recordings, the signals were bandpass filtered to 0.03$300 \mathrm{~Hz}$ and digitized with a sampling frequency of 1 $\mathrm{kHz}$.

In the BSPM measurements, unipolar potentials were recorded at 120 locations covering the whole thorax. ${ }^{20}$ In addition, three limb leads were recorded with electrodes on the right and left shoulder and the left hip. Wilson central terminal was used as a reference for the unipolar leads. The $120 \mathrm{Ag} / \mathrm{AgCl}$ electrodes were mounted on 18 strips with an interelectrode distance of $5 \mathrm{~cm}$. The strips were placed on the subject's thorax vertically and their horizontal spacing was determined using the dimensions of the upper body. Altogether 45 of the electrodes were on the posterior thorax. The front-end electronics of the mapping system was placed inside the MSR, and the recorded data were transferred optically to a personal computer controlling the data acquisition outside the MSR. After bandpass filtering from 0.16 to $300 \mathrm{~Hz}$, the signals were digitized with the sampling rate of $1 \mathrm{kHz}$.

Physical stress was induced using an exercise ergometer constructed specially for magnetocardiographic measurements. The ergometer was composed of nonmagnetic materials, and induces no notable magnetic fields. The ergometer is pedaled in a supine position, and different load levels can be applied.

The center of the cardiomagnetometer was placed 15 $\mathrm{cm}$ below the jugulum and $5 \mathrm{~cm}$ left of the midsternal line (Fig. 1). Distance from the chest was set to enable maximal inhalation without a contact between skin and the bottom of the magnetometer. Simultaneously with the MCG, the 12-lead ECG was recorded. After a measurement at rest for $5 \mathrm{~min}$, exercise was started and the load was increased every $2 \mathrm{~min}$. The cessation criteria for the stress were as recommended for the standard exercise ECG testing. ${ }^{5}$ After exercise, the measurement was continued for 10 min during recovery.

The MCG and BSPM measurements were performed separately during the same day. In one half of the subjects the MCG was recorded first. In both recordings, the same exercise protocol was used. The same three limb lead reference electrodes were used both in the MCG and BSPM.

\section{Signal Processing}

MCG and BSPM data at rest and immediately after the cessation of exercise were selectively averaged. First, channels with excessive noise level were excluded from further analysis. A template beat of raw data with good signal quality in visual observation was then selected. In order to establish the baseline level, a regression line was fitted on two sets of data points, selected at a PQ interval and at the next TP interval on each channel. In the case of high heart rate with nondetectable TP interval, two consecutive PQ intervals were used. To cancel linear baseline shift the line was subtracted from the signal. The baseline-corrected beats of raw data were then compared with the template. A range of acceptable data values, called a tube, was formed around the template. The tube was calculated by shifting the template both in time and amplitude directions resulting in envelope curves above and below the template. ${ }^{15,21}$ The shift in time was $\pm 5 \mathrm{~ms}$. To reject artifacts, the template was compared with the first cardiac cycle of raw data and if the candidate beat did not fit inside the tube it was excluded from the average. The cumulative average was used as the template for the next candidate beat. Both at rest and after the cessation of exercise, 40 cardiac cycles of raw 


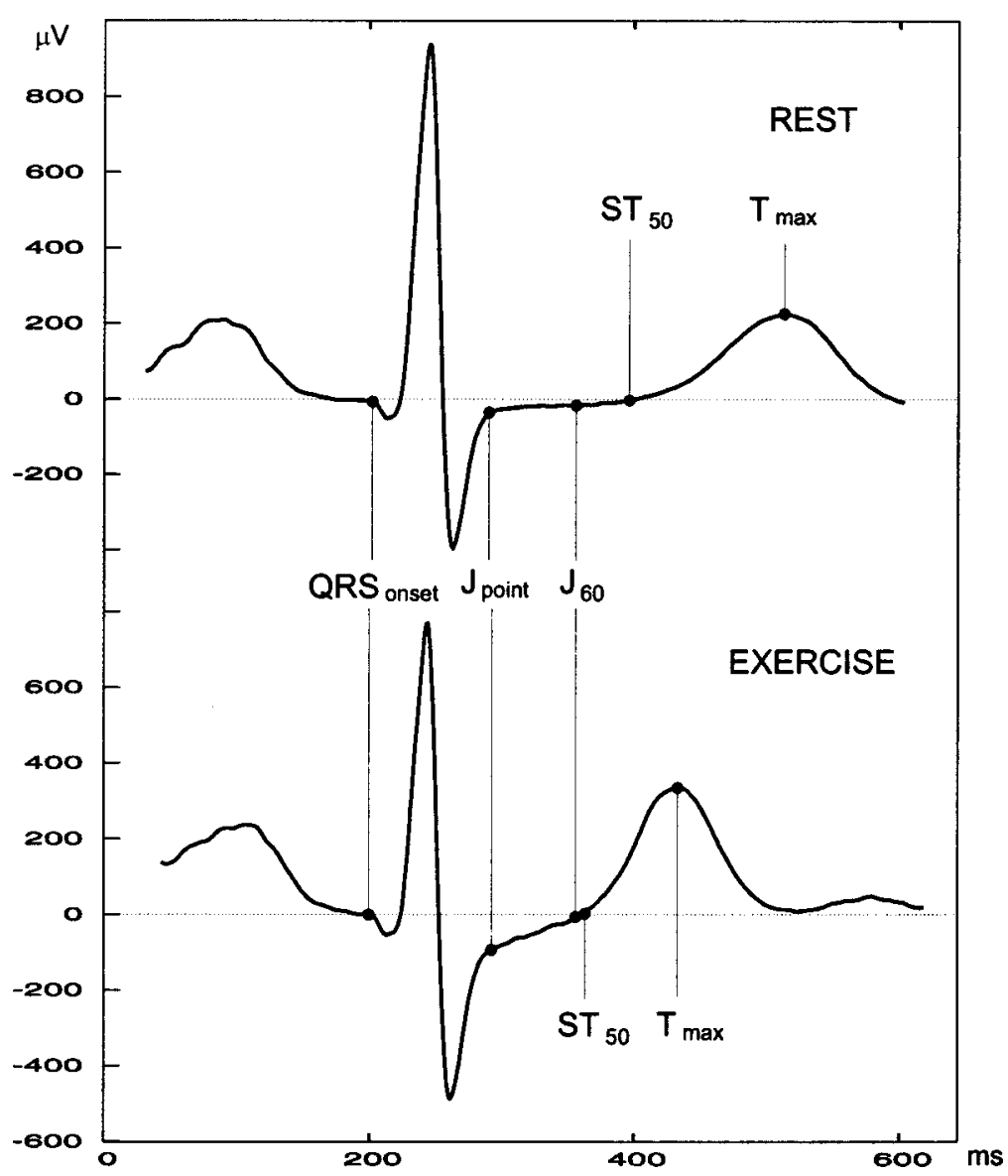

FIGURE 2. Signal of ECG lead II recorded at rest (above) and immediately after exercise (below), showing the time instants selected for the evaluation. In the ST segment, $\mathrm{ST}_{50}$ is in the middle of $J_{\text {point }}$ and $T_{\max }$, while $J_{60}$ is $60 \mathrm{~ms}$ after the $J_{\text {point }}$.

data were processed. In calculating the envelope curves, the amplitude shift of the template was adjusted to include approximately one half of the candidate beats in the average. If, e.g., less than 20 beats were included in the average the tube was broadened and the averaging was done over.

Five time points of cardiac cycle in the MCG and BSPM were determined using the signal-averaged data of the ECG lead II (Fig. 2). These time points were: (1) the QRS onset, (2) the QRS offset $\left(J_{\text {point }}\right)$, (3) $60 \mathrm{~ms}$ from the $J_{\text {point }}$ into the ST segment $\left(J_{60}\right)$, (4) T-wave apex $\left(T_{\max }\right)$, and (5) the mid point of $J_{\text {point }}$ and $T_{\max }$ $\left(\mathrm{ST}_{50}\right)$. Two time instants of the ST segment were thus selected for evaluation; $J_{60}$ fixed in time and $\mathrm{ST}_{50}$ fixed in signal morphology.

To make the signals of the two types of gradiometers comparable, the signal-averaged 67-channel MCG data were converted to correspond to data of 33 coaxial gradiometers. Here, we used minimum-norm estimation of the source current distribution. ${ }^{3,13}$ The obtained virtual sensors were located at the sites of planar and axial gradiometer units in the cardiomagnetometer (see the Appendix).

Three amplitude values $\left(J_{60}, \mathrm{ST}_{50}\right.$, and $\left.T_{\max }\right)$ were measured on each channel of both MCG and BSPM data at rest and at the cessation of exercise. Also, the average signal from QRS onset to QRS offset (QRS integral) was determined on each channel. For quantification of the signal amplitude, the minimum and maximum signal values were determined in each subject and in each time point.

The range of the QRS complex in the MCG mapping was calculated as the difference between the highest and the lowest signal value detected over the mapped area from the QRS onset to the QRS offset (QRS range). In a similar way, the ST-segment range was calculated be-

TABLE 1. Amplitude ranges of QRS complex and ST segment at rest and at the cessation of exercise in the MCG and BSPM.

\begin{tabular}{lcc}
\hline & \multicolumn{2}{c}{$\begin{array}{c}\text { Mean } \pm \text { SD over individuals } \\
\text { within the study group }\end{array}$} \\
\cline { 2 - 3 } & QRS range & ST range \\
\hline MCG rest $(\mathrm{pT})$ & $37.3 \pm 7.9$ & $3.4 \pm 2.0$ \\
MCG exercise $(\mathrm{pT})$ & $33.6 \pm 8.2^{\mathrm{a}}$ & $4.5 \pm 1.9^{\mathrm{b}}$ \\
BSPM rest $(\mu \mathrm{V})$ & $3320 \pm 550$ & $420 \pm 90$ \\
BSPM exercise $(\mu \mathrm{V})$ & $3290 \pm 850$ & $430 \pm 130$ \\
\hline
\end{tabular}

${ }^{\mathrm{a}} p=0.005$ vs rest.

${ }^{\mathrm{b}} p=0.008$ vs rest. 
TABLE 2. The highest and the lowest amplitudes detected at the time points evaluated.

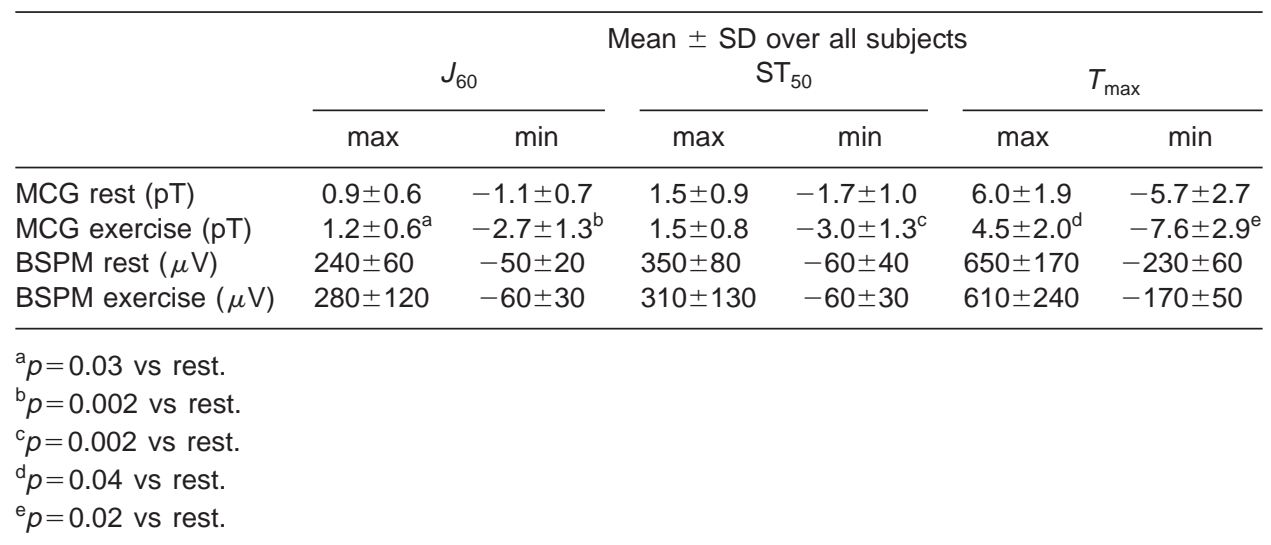

tween the $J_{\text {point }}$ and the $\mathrm{ST}_{50}$ (ST range). The corresponding electric potential ranges were calculated using the BSPM data.

For the illustration of spatial signal distributions, both the MCG and the BSPM isocontour maps were interpolated from the data using minimum-norm estimation ${ }^{3,13}$ (see the Appendix). Also, to characterize the field distribution in the whole study group, MCG and BSPM group-mean maps were calculated as the mean of individual maps.

A group-mean map characterizes the spatial features of the signal distribution as an average over all subjects in the study group. To quantify the variance in the distributions within the group, the correlation of signal values in each individual map with the values in the groupmean map was calculated. Here, the Pearson productmoment correlation was used. The capability of the group-mean map to describe the field pattern of the whole study group was quantified with the mean of the correlations.

To evaluate the effect of exercise, individual and group-mean difference maps were formed by subtracting the spatial MCG and BSPM distributions at rest from the corresponding distributions immediately after exercise. The alterations in the signal distributions from rest to the cessation of exercise were quantified by calculating the correlations of the MCG and BSPM maps at rest with the corresponding maps at the cessation of exercise. The calculation was performed in each subject and in all time points selected for evaluation. The degree of change due to exercise in the whole study group was quantified as the mean of the correlations. Also, the correlation of the group-mean map at rest with the group-mean map at the cessation of exercise was calculated to assess the effect of exercise.

The significance level of difference between the parameters calculated at rest and at the cessation of exercise was defined with Wilcoxon signed ranks test for two dependent samples. Values $p<0.05$ were considered significant.

\section{RESULTS}

Heart Rate

On the average, the heart rate in the MCG measurements increased from $65 \pm 11$ beats per minute (bpm) at rest to $127 \pm 14 \mathrm{bpm}$ after exercise, and in the BSPM measurements from $69 \pm 10$ to $134 \pm 16 \mathrm{bpm}$. The difference in the maximum heart rates reached in the MCG and BSPM recordings was not significant $(p=0.3)$.

\section{Signal Ranges}

The mean QRS-complex and ST-segment ranges over all subjects at rest and at the cessation of exercise are presented in Table 1 for the MCG and BSPM. The MCG signal range of the QRS complex was lower and the ST-segment range higher at the cessation of exercise compared with values at rest $(p=0.005$ and $p=0.008$, respectively).

\section{Signal Amplitudes}

The highest and the lowest MCG and BSPM signal values within the mapped area are presented in Table 2. In the BSPM, the alterations in potential values from rest to the cessation of exercise were not significant. In the MCG, an amplification was found in the negative field extremum in all three time points evaluated. Also, an increase at $J_{60}$ and a decrease at $T_{\max }$ took place in the highest MCG signal value over the mapped area (Table 2).

\section{Spatial Patterns}

QRS Complex. The MCG and BSPM group-mean maps of QRS integral and the corresponding group-mean dif- 


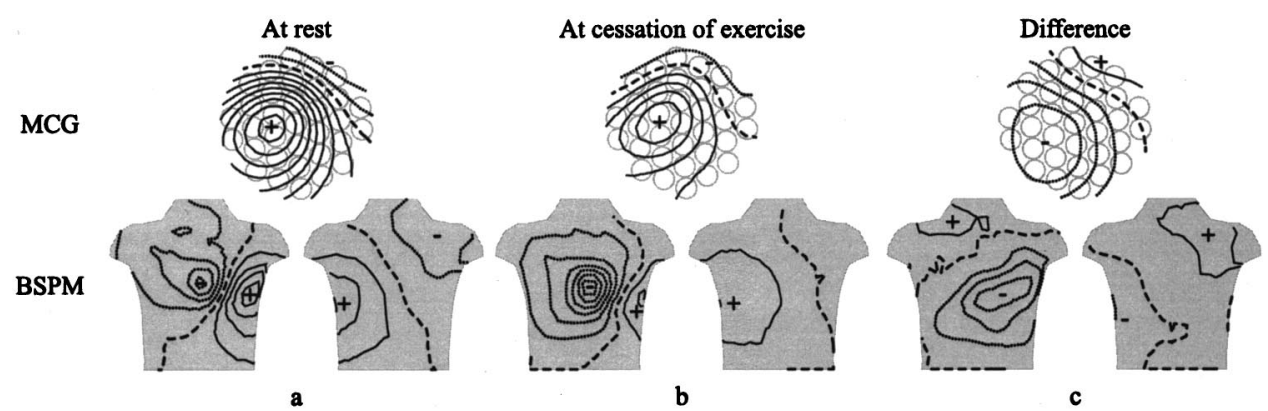

FIGURE 3. Group-mean isointegral maps of the QRS complex: MCG (above) and BSPM (below) (a) at rest and (b) at the cessation of exercise. (c) The change in the maps from rest to the cessation of exercise. The steps between two consecutive lines are $0.5 \mathrm{pT}$ (MCG), and $50 \mu \mathrm{V}$ (BSPM). Solid lines indicate positive and dotted lines negative field or potential. Broken curve indicates the zero signal. In the MCG maps, positive values indicate magnetic flux towards the chest. In the potential maps, torso silhouettes represent the potential over the anterior chest (left), and over the back (right).

ference maps are presented in Fig. 3. The spatial patterns of the BSPM maps were almost similar at rest and at the cessation of exercise, but the potential values decreased over the anterior chest. The correlations of individual maps with the group-mean map were, on the average, 0.84 at rest and 0.76 at the cessation of exercise.

In the MCG, the field maximum in the group-mean QRS integral map was found at the same location before and after exercise (Fig. 3). The spatial pattern of the group-mean integral map at the cessation of exercise differed from that at rest, but the individual variation in the maps was extensive; the correlation of the individual MCG maps with the group-mean map was on the average 0.71 at rest and only 0.44 after exercise.

In the group-mean difference maps (Fig. 3), the negative MCG and BSPM values indicate a decrease in the integral value over most of the mapped area due to exercise. In the individual BSPM maps, both the largest and the smallest integral value were lower after exercise when compared to rest $(p<0.005)$. In the MCG, the maximum integral value within the mapped area was smaller at exercise than at rest $(p=0.003)$, but the value of the integral minimum did not change significantly.
ST Segment. The MCG and BSPM group-mean distributions at $J_{60}$ are presented in Fig. 4. The BSPMs at rest showed a potential maximum over the precordium. The positive potential extended over the anterior thorax, while the right superior and the posterior parts of the thorax had a weak negative potential. After exercise, the signal patterns showed only small changes when compared to maps at rest. Mean correlations of individual maps with the group-mean maps at rest and after exercise were 0.91 and 0.86 , respectively.

In spite of considerable individual variation in the rest MCG maps at $J_{60}$, a tendency of a positive field extremum on the inferior half and negative extremum on the superior half of the mapped area was observed (Fig. 4). The amplitudes of both the highest and the lowest signal values increased from rest to the cessation of exercise (Table 2). Mean correlations of individual maps with the group-mean maps were lower than in the BSPM: 0.70 at rest and 0.80 at the cessation of exercise, indicating higher variance in the spatial signal patterns within the study group.

Both BSPM and MCG group-mean distributions at $\mathrm{ST}_{50}$ (Fig. 5) were almost similar as the corresponding
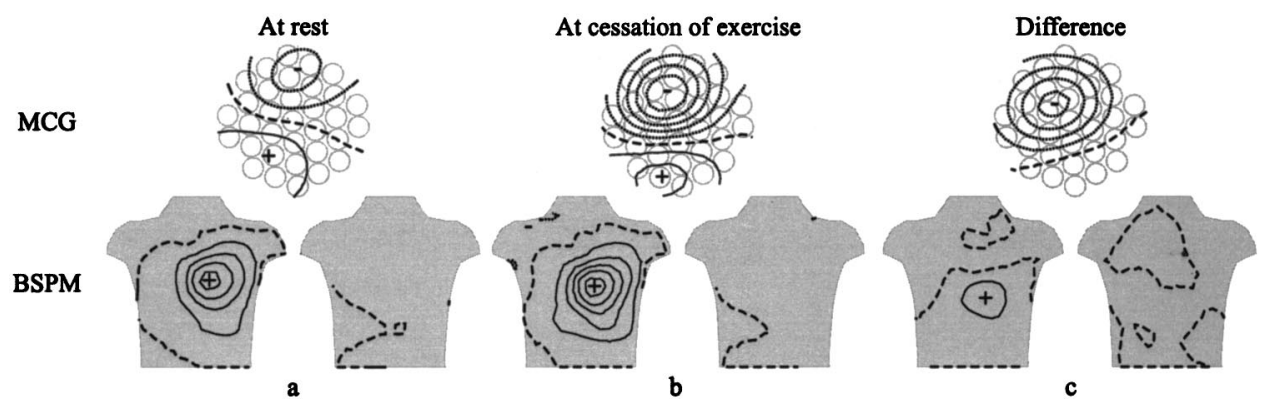

FIGURE 4. Group-mean maps at $J_{60}$ : MCG (above) and BSPM (below) (a) at rest and (b) after exercise. (c) The difference maps. In the MCG maps the step between two consecutive isofield lines is $0.5 \mathrm{pT}$, and in the BSPM $50 \mu \mathrm{V}$. The format is the same as in Fig. 3. 


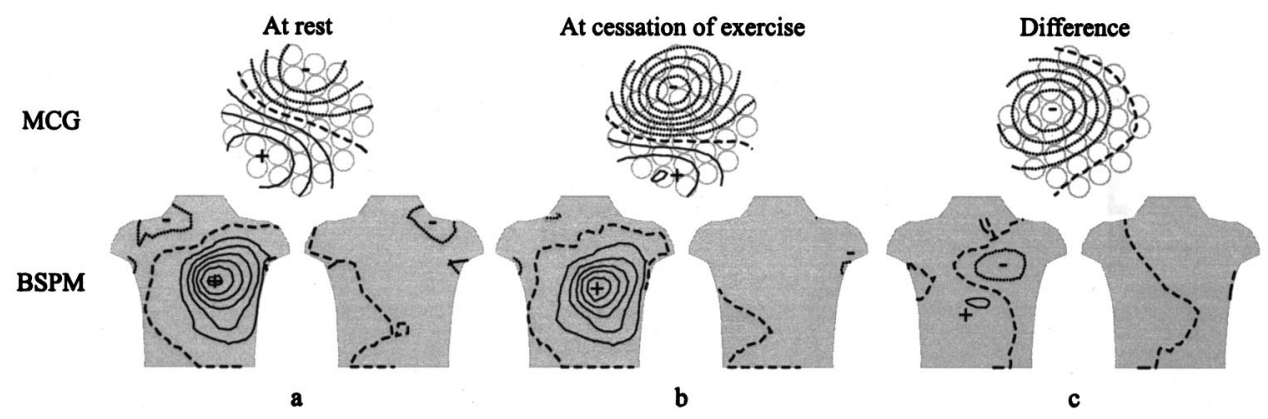

FIGURE 5. Group-mean maps at $\mathrm{ST}_{50}$ : MCG (above) and BSPM (below) (a) at rest and (b) at the cessation of exercise. (c) The difference maps. In the MCG maps the step between two consecutive isofield lines is 0.5 pT, and in the BSPM $50 \mu$ V. The format is the same as in Fig. 3.

maps at $J_{60}$. A dominant BSPM maximum was detected over the precordium at rest and at the cessation of exercise. The BSPM group mean difference distribution indicated small alteration from rest to the cessation of exercise. In contrast, relatively high negative values in the MCG group-mean difference map showed a depression in the signal due to exercise. The mean correlation of individual maps with the group-mean maps was higher in BSPM than in MCG, both at rest $(0.92$ and 0.79 , respectively) and at exercise $(0.88$ and 0.82 , respectively).

T wave. The group-mean BSPM maps both at rest and at the cessation of exercise were dominated by a positive area over the precordium and lower left thorax at $T_{\max }$ (Fig. 6). Potential minimum was located over the upper right part of the torso, and the negative potential extended over the superior and right lateral thorax. In the group-mean difference map, a minor decrease in potential over the superior precordium and an increase over the inferior precordium were detected from rest to exercise. The correlation of individual isopotential maps with the group-mean map was high both at rest and at exercise (mean 0.95 and 0.94 , respectively), indicating low individual variation in the maps.
The group-mean MCG maps at $T_{\max }$ had a maximum both at rest and after stress over the lower right half of the mapped area (Fig. 6). The corresponding minima were located over the upper left part of the mapped area; the field patterns were thus approximately perpendicular with the BSPM group-mean maps. A shift and bending of the spatial zero field line downwards and to the right took place after stress when compared to rest. A decrease in the MCG signal values from rest to the cessation of exercise was detected in the difference maps. The groupmean difference map showed a monopolar field pattern over the mapped area. The mean correlations of individual maps with the group-mean maps were 0.92 at rest and 0.90 at exercise.

\section{Correlation of MCG and BSPM Maps at Rest with Maps at Exercise}

The mean correlations of individual MCG and BSPM maps at rest with the corresponding individual maps at the cessation of exercise are presented in Table 3. The correlation at the ST segment and T wave was, on the average, higher in BSPM than in MCG, but the difference was not significant.
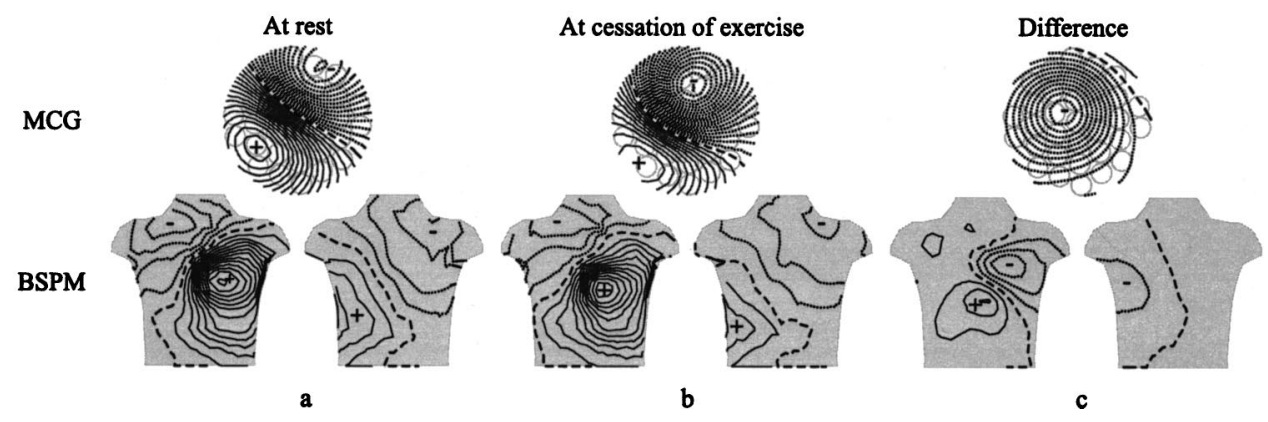

FIGURE 6. Group-mean maps at $T_{\max }$ : MCG (above) and BSPM (below) at (a) at rest and (b) at the cessation of exercise. (c) The difference maps. In the MCG maps the step between two consecutive isofield lines is $0.5 \mathrm{pT}$, and in the BSPM $50 \mu \mathrm{V}$. The format is the same as in Fig. 3. 
TABLE 3. The mean correlations of individual magnetic field and electric potential maps at rest with the corresponding maps at the cessation of exercise.

\begin{tabular}{lcccc}
\hline & QRS $_{\text {integral }}$ & $J_{60}$ & $\mathrm{ST}_{50}$ & $T_{\max }$ \\
\hline MCG & $0.78 \pm 0.36$ & $0.70 \pm 0.30$ & $0.74 \pm 0.27$ & $0.84 \pm 0.14$ \\
BSPM & $0.77 \pm 0.20$ & $0.88 \pm 0.15$ & $0.89 \pm 0.13$ & $0.93 \pm 0.04$ \\
\hline
\end{tabular}

In Table 4, the correlations of the group-mean MCG and BSPM maps at rest with the corresponding groupmean maps at exercise are presented for all time points evaluated. During the ST segment and T wave, the correlation was lower in the MCG maps than in the BSPM maps, indicating larger change due to exercise in the MCG. In the QRS-complex integral maps, the correlation was higher in the MCG than in the BSPM.

\section{DISCUSSION}

In this paper we present a comparison of multichannel magnetocardiography with body surface potential mapping over the whole thorax in middle-aged healthy subjects during exercise testing. The changes in spatial signal distribution due to exercise were relatively small and the correlation coefficients between maps recorded at rest and after exercise were of the same order in the MCG and the BSPM. In the signal amplitude, however, a more extensive change took place in the MCG compared to the BSPM. The signal minimum at the ST segment and at the $\mathrm{T}$ wave amplified in the MCG due to exercise. A corresponding change was not observed in the BSPM. The spatial pattern of the change in the MCG indicated a magnetic flux component outward of the subject's thorax over the mapped area.

The MCG is a measurement of a vector field quantity, whereas in the BSPM the electric potential is recorded. Also the measurement geometries are different in the two methods. Therefore, the extent of change induced by exercise in the MCG and BSPM signals cannot be compared directly. We calculated the statistical significance of alteration due to exercise in the MCG and BSPM separately. Thereafter, we compared the significance levels and found the MCG signal to be amplified more due to exercise than the BSPM signal.

Brockmeier et al. reported ST-T segment changes in three healthy subjects in multichannel MCG after phar-

TABLE 4. Correlations of magnetic field and electric potential group-mean maps at rest with the corresponding maps at the cessation of exercise.

\begin{tabular}{lcccc}
\hline & QRS $_{\text {integral }}$ & $J_{60}$ & ST $_{50}$ & $T_{\max }$ \\
\hline MCG & 0.95 & 0.82 & 0.81 & 0.92 \\
BSPM & 0.73 & 0.95 & 0.94 & 0.95 \\
\hline
\end{tabular}

macological stress, not visible in 32-lead ECG. These changes included ST-segment depression as well as T-wave inversion with monopolar negative MCG maps after stress. The authors suggested that these changes were induced by vortex currents, not detectable by the ECG. $^{2}$ The findings of the present work with a larger study population and more extensive spatial sampling in the BSPM support the results by Brockmeier et al. The monopolar MCG pattern found in the difference maps might be induced by a circular current. Differences between the work presented earlier ${ }^{2}$ and this study in the MCG field patterns after exercise could originate from the different measurement setups. In particular the thoracic area covered by the cardiomagnetometer is likely to affect the correlation coefficients calculated. Comparison of exercise data recorded with both measurement setups and transformed to the other configuration is needed to evaluate this hypothesis.

A tendency to QRS potential range decrease and STsegment range increase due to exercise was found in the BSPM. These results support the earlier findings by McPherson et al. ${ }^{10}$ in upright stress testing. In addition, more extensive alterations were found in the MCG than in the BSPM; a more significant decrease took place in the signal range over the QRS complex, and a more evident increase was found in the ST-segment range in the MCG than in the BSPM.

Contrary to the BSPM, the MCG covers a limited area of the thorax. Some significant features of the magnetic field, e.g., the positive signal extremum, may therefore be situated outside the mapped area. In this work with MCG covering a limited area over the anterior chest, the signal minimum was amplified at the ST segment and the $\mathrm{T}$ wave due to exercise. We do not expect this amplification to vanish by extension of the mapping area since the great majority of the maps evaluated had a negative signal extremum over the mapped area both at rest and after stress. The difference maps had a clear negative extremum over the mapped area in all subjects and time instants evaluated. Since the MCG signal has the highest amplitude over the precordium it is unlikely that a more extensive amplitude change due to exercise will be found elsewhere.

The individual variation in the MCG and BSPM maps was quantified with the mean correlation between individual maps and the group-mean map. The variation was lowest at the T-wave apex, where the signal amplitude is also higher than at the ST segment. Therefore, the groupmean maps of the $\mathrm{T}$ wave, presented here have a higher value as reference data than the maps of the ST segment. In the QRS complex, both negative and positive deflections contribute to the integral value, partially canceling each other. This makes the integral map sensitive to the morphology of the QRS complex and could explain the 
high variation in the integral maps within the study group.

\section{LIMITATIONS OF THE STUDY}

The study population in this work was small and only one female subject was included. Therefore, evaluation of gender based differences between the MCG and BSPM would require more recordings, especially with female subjects. Technical limitations prevented the simultaneous recordings of the MCG and the 123-lead BSPM, which would be the optimal arrangement for a comparison of the two mappings. The two exercise recordings were, however, performed in random order and heart rates at peak exercise did not differ significantly. Also, the time intervals for evaluation were selected using the ECG lead II which was recorded with the same electrodes in both measurements.

\section{CONCLUSION}

In the development of magnetocardiographic methods for the detection of exercise-induced ischemia, the precordial negative field component during the repolarization period of the cardiac cycle should be considered as a normal response to physical exercise. Therefore, magnetocardiographic evaluation of myocardial ischemia should not be based solely on ST-segment amplitude changes used in the standard 12-lead ECG. Also the changes of spatial field patterns should be taken into account. The present study provides reference data for future studies on exercise MCG.

\section{ACKNOWLEDGMENTS}

This work was supported by the Jenny and Antti Wihuri Foundation, The Foundation of Technology in Finland, and the Academy of Finland.

\section{APPENDIX: INTERPOLATION WITH MINIMUM- NORM ESTIMATION}

The 33-channel axial gradiometer signals and the isocontour (MCG and BSP) maps and were interpolated from the measured data using minimum-norm estimation (MNE). ${ }^{7} \mathrm{MNE}$ is a current density field $\mathbf{J}^{*}$ defined as a linear combination of the lead fields of the MCG coils or BSPM electrodes, $L_{k}: \mathbf{J}^{*}(\mathbf{r})=\sum_{k=1}^{N} \omega_{k} L_{k}(\mathbf{r})$. It is the current distribution that has the smallest norm and is compatible with the measured data. We define the next matrix $\Gamma$ consisting of inner products between the lead fields of all sensor pairs $L_{i}$ and $L_{j}$. Then, the weighting coefficients $\omega_{k}$ are found from the measured MCG or BSPM signals $b^{m}$ by $\omega_{k}=\Gamma^{-1} b^{m}$. Since the lead fields in a large array are almost linearly dependent, regularization techniques are needed to produce stable estimates. $^{7}$

Interpolation with MNE is based on evaluating the lead fields of virtual sensors, $L_{k}^{s}$, and composing matrix $\Gamma^{\prime}$ from the dot products between measurement and virtual lead fields. The interpolated signals are then obtained as $b^{e}=\Gamma^{\prime} \Gamma^{-1} b^{m} \cdot{ }^{3,13}$ For isocontour and axial gradiometer interpolation, the sensor array surface was triangulated. The field component $B_{z}$ perpendicular to the surface was interpolated at each time instant by assuming a virtual point sensor at each node on the triangulated surface. Axial gradiometers $\left(\partial B_{z} / \partial z\right)$ were interpolated by placing a virtual axial sensor at each true sensor point. For the BSPM, the corresponding potential value interpolation was performed on the nodes of the standard torso model.

\section{REFERENCES}

${ }^{1}$ Brockmeier, K., S. Comani, S. Erne, A. Di Luzio, A. Pasquarelli, and G. L. Romani. Magnetocardiography and exercise testing. J. Electrocardiol. 27:137-142, 1994.

${ }^{2}$ Brockmeier, K., L. Schmitz, J. Chavez, M. Burghoff, H. Koch, R. Zimmerman, and L. Trahms. Magnetocardiography and 32-lead potential mapping: Repolarization in normal subjects during pharmacologically induced stress. J. Cardiovasc. Electrophysiol. 8:615-626, 1997.

${ }^{3}$ Burghoff, M., J. Nenonen, L. Trahms, and T. Katila. Conversion of magnetocardiographic recordings between two different multichannel SQUID devices. IEEE Trans. Biomed. Eng. 47:869-875, 2000.

${ }^{4}$ Cohen, D., P. Savard, R. D. Rifkin, E. Lepeschkin, and W. E. Strauss. Magnetic measurement of S-T and T-Q segment shifts in Humans. Part II: Exercise-induced S-T segment depression. Circ. Res. 53:274-279, 1983.

${ }^{5}$ Fletcher, G., G. Balady, V. Froelicher, L. Hartley, W. L. Haskell, and M. L. Pollock. Exercise standards. A statement for healthcare professionals from the American Heart Association. Circulation 91:580-615, 1995.

${ }^{6}$ Hailer, B., P. Van Leeuwen, S. Lange, and M. Wehr. Spatial distribution of QT dispersion measured by magnetocardiography under stress in coronary artery disease. J. Electrocardiol. 32:207-216, 1999.

${ }^{7}$ Hämäläinen, M. S., and J. Nenonen. Magnetic source imaging. In: Encyclopedia of Electrical Engineering, edited by J. G. Webster. New York: Wiley, 1999, pp. 133-148.

${ }^{8}$ Hänninen, H., P. Takala, M. Mäkijärvi, J. Montonen, P. Korhonen, L. Oikarinen, J. Nenonen, T. Katila, and L. Toivonen. Detection of exercise-induced myocardial ischemia by multichannel magnetocardiography in single vessel coronary artery disease. Ann. Noninv. Electrocardiol. 5:147-157, 2000.

${ }^{9}$ Lant, J., G. Stroink, B. ten Voorde, B. M. Horacek, and T. J. Montague. Complementary nature of electrocardiographic and magnetocardiographic data in patients with ischemic heart disease. J. Electrocardiol. 23:315-322, 1990.

${ }^{10}$ McPherson, D., M. B. Horacek, D. J. Sutherland, C. S. Armstrong, C. A. Spencer, and T. J. Montague. Exercise electro- 
cardiographic mapping in normal subjects. J. Electrocardiol. 18:351-360, 1985.

${ }^{11}$ Montonen, J., A. Ahonen, M. Hämäläinen, R. J. Ilmoniemi, P. Laine, J. Nenonen, M. Paavola, K. Simelius, J. Simola, and T. Katila. Magnetocardiographic functional imaging studies in the BioMag laboratory. In: Biomag 96, Proceedings of the Tenth International Conference on Biomagnetism, edited by C. J. Aine, Y. Okada, G. Stroink, S. J. Swithenby, and C. C. Woods. New York: Springer, 2000, pp. 494-497.

${ }^{12}$ Moshage, W., and S. Achenbach. Clinically significant differences between ECG and MCG. Biomed. Tech. 42:25-27, 1997.

${ }^{13}$ Numminen, J., S. Ahlfors, R. Ilmoniemi, J. Montonen, and J. Nenonen. Transformation of multichannel magnetocardiographic signals to standard grid form. IEEE Trans. Biomed. Eng. 42:72-78, 1995.

${ }^{14}$ Paavola, M., R. Ilmoniemi, L. Sohlström, T. Meinander, A. Penttinen, and T. Katila. High performance magnetically shielded room for clinical measurements. In: Biomag 96, Proceedings of the Tenth International Conference on Biomagnetism, edited by C. J. Aine, Y. Okada, G. Stroink, S. J. Swithenby, and C. C. Woods. New York: Springer, 2000, pp. 87-90.

${ }^{15}$ Paavola, M., K. Simelius, J. Montonen, J. Nenonen, and T. Katila. Averaging in Cardiographic Signal Processing. In: Finnish Signal Processing Symposium, Finsig'95, Symposium Proceedings. Espoo: TKK Offset, 1995, pp. 138-141.

${ }^{16}$ Roth, B., and J. Wikswo. Electrically silent magnetic fields. Biophys. J. 50:739-745, 1986.

${ }^{17}$ Saarinen, M., P. Karp, T. Katila, and P. Siltanen. The magnetocardiogram in cardiac disorders. Cardiovasc. Res. 8:820834, 1974.
${ }^{18}$ Seese, B., W. Moshage, S. Achenbach, R. Killmann, and K. Bachmann. Magnetocardiographic (MCG) analysis of myocardial injury currents. In: Biomagnetism: Fundamental Research and Clinical Applications, edited by C. Baumgartner, C. Deecke, G. Stroink, and S. J. Williamson. Amsterdam: Elsevier/IOS, 1995, pp. 628-632.

${ }^{19}$ Siltanen, P. Magnetocardiography. In: Comprehensive Electrocardiology, edited by P. W. MacFarlane and T. D. Veitch Lawrie. New York: Pergamon, 1989, pp. 1405-1438.

${ }^{20}$ Simelius, K., I. Tierala, T. Jokiniemi, J. Nenonen, L. Toivonen, and T. Katila. A body surface potential mapping system in clinical use. Med. Biol. Eng. Comput. 34:107-108, 1996.

${ }^{21}$ Väänänen, H., P. Korhonen, J. Montonen, M. Mäkijärvi, J. Nenonen, L. Oikarinen, K. Simelius, L. Toivonen, and T. Katila. Non-invasive arrhythmia risk evaluation in clinical environment. Herzschr. Elektrophys. 11:229-234, 2000.

${ }^{22}$ Van Leeuwen, P., G. Stroink, B. Hailer, A. Adams, S. Lange, and D. Grönemeyer. Rest and stress magnetocardiography in coronary artery disease. Med. Biol. Eng. Comput. 37:14821483, 1999.

${ }^{23}$ Wikswo, J. P. Theoretical aspects of the ECG-MCG relationship. In: Biomagnetism, An Interdisciplinary Approach, edited by S. Williamson, G-L. Romani, L. Kaufman, and I. Modena. New York: Plenum, 1983, pp. 311-326.

${ }^{24}$ Yanowitz, F., G. Vincent, R. Lux, M. Merchant, L. S. Green, and J. A. Abildskov. Application of body surface mapping to exercise testing; ST-isoarea maps in patients with coronary artery disease. Am. J. Cardiol. 50:1109-1113, 1982. 